\title{
Erratum
}

\section{Symmetry-adapted integral derivatives}

\author{
Peter R. Taylor* \\ ELORET Institute, Sunnyvale, CA 94087, USA \\ Theor Chim Acta (1986) 69:447
}

(Received March 24, 1989)

In the above paper there is an error in the form of the selection rule used to identify vanishing integral derivatives. Throughout the paper, Kronecker delta factors of the form $\delta_{R(B), E}$, etc. should be replaced by $\delta_{B E}$, that is, the symmetry operation $R$ should not appear. There is then an additional factor of $p_{\sigma}(R)$ to be included with each $\delta_{B E}$ in the special case of $D_{2 h}$ and its subgroups only (Eqs. $(50)-(56)$ ). The parity factor $p_{\sigma}(R)$ (see Sect. 4) is defined by the Cartesian direction $\sigma$ with respect to which differentiation takes place. Note that the phase factors $\Lambda_{\tilde{\sigma} \sigma \bar{m} v}^{R(B) \epsilon}$ in the general formulas from which these special cases are derived are correct.

In addition, it should be noted that some care is required in interpreting the phase factors arising from the use of translational invariance, e.g. Eq. (53). Symmetry operations may invert the sense of the Cartesian basis vectors and it will depend on a particular computational implementation of symmetry whether these factors need be included explicitly or not.

Finally, there are several typographical errors in the paper, notably the summation variables in Eqs. (5) and (6), both of which should be $\bar{a}$, and in the integrals appearing in Eq. (50), where the basis functions should be $f_{b R(B)}$ and $f_{b R(B)}^{\sigma}$.

These errors came to light during the development of a symmetry-adapted integral derivative code [1], and I would like to thank Trygve Helgaker for many helpful discussions.

\section{References}

1. Helgaker T, Jensen HJAa, Jørgensen P, Taylor PR: ABACUS: a symmetry-adapted MCSCF properties program

\footnotetext{
*Mailing address: NASA Ames Research Center, Moffett Field, CA 94035, USA
} 